\title{
Ray-tracing-based time-domain wideband fractional delay beamforming for underwater acoustic positioning
}

\author{
Haipeng $\mathrm{Li}^{1,2,3, *}$, Qingdong Yuan ${ }^{4}$, Yingyuan $\mathrm{Wu}^{4}$, and Xuebin $\mathrm{Wang}^{4}$ \\ ${ }^{1}$ Acoustic Science and Technology Laboratory, Harbin Engineering University, Harbin 150001, China; \\ ${ }^{2}$ Key Laboratory of Marine Information Acquisition and Security (Harbin Engineering University), Ministry of Industry and \\ Information Technology, Harbin 150001, China \\ ${ }^{3}$ College of Underwater Acoustic Engineering, Harbin Engineering University, Harbin 150001, China \\ ${ }^{4}$ CNOOC Deepwater Development Limited, Shenzhen 518000, China
}

\begin{abstract}
Underwater acoustic positioning systems are essential for underwater vehicles positioning and navigation. In this paper, a time-domain wideband fractional delay beamforming (WFDB) technique is proposed to improve underwater localization capability of planar arrays. In our method, digital delay lines are combined with adaptive-finite-impulse-response filters to achieve high accuracy beamforming results and reduce the design burden of the WFDB systems. Further, the beamforming results are used in the raytracing method to eliminate curvature effect of sound rays caused by the inhomogeneity of water, and therefore improving the computation accuracy of target position. We further develop a hexagonal planar array of 30 elements to verify the WFDB method in an indoor experimental pool. The experimental results demonstrate that the proposed method could successfully mitigate the ray bending problem and accurately estimate the position of targets in low SNR condition.
\end{abstract}

\section{Introduction}

The sound wave is the most effective carrier for underwater information transmission [1,2]. Underwater acoustic positioning systems (UAPSs) are regarded as essential positioning and navigation components for underwater vehicles [3-6]. UAPSs, in which a sonar array is employed to determine the range and bearing to the vehicles, are indeed routinely used today [7]. Compared with the linear array, the planar array has better spatial angular resolution. It can not only determine the pitch angle of the direction-of-arrival (DOA), but also determine the azimuth angle.

Conventional beamforming $(\mathrm{CBF})$ is a widely used method for array signal processing [8]. The received signals are delayed and summed based on the relationship between the configuration of sensors and the incident angle of the signal [9]. When the steering angle is aligned with the incident angle of the signal, the signals are coherently summed while the noise are not (the noise is assumed uncorrelated) which resulting in a higher signal-to-noise radio (SNR) at the output compared with the input SNR[10].

Sound velocity in the sea changes with the depth [1]. Sound velocity bias is the primary cause of position error in the UAPSs over a long range. Thus, the unique characteristics of underwater acoustic channels necessitate a sound velocity correction algorithm which properly accounts for sound trajectory bending. raytracing is the most commonly used method for the problem. In ray-tracing theory, the sound trajectory consists of many arcs under the assumption of a stratified sound velocity gradient [11]. It makes use of standard ray equations to model the sound trajectories in water and could achieve more accurate results as compared with other methods $[12,13]$.

In this paper, we propose a ray-tracing-based timedomain wideband fractional delay beamforming for UAPSs. We combine digital delay line with adaptive FIR filter in CBF, where the beamforming accuracy can be ensured, and the design burden of the beamforming systems can be reduced. Further, we apply the beamforming result as an initial grazing angle for raytracing to eliminate the curvature effect of the sound trajectory caused by the inhomogeneity of water, which is the main factor that influences the computation accuracy of target position. Moreover, a hexagonal planar array (UHL) of 30 elements is developed to verify our method algorithm. The experimental results show that, the proposed method can effectively reduce the effect of ray bending and accurately estimate the position of target in low SNR.

\section{DOA estimation via conventional beamforming (CBF)}

CBF is a well-known array processing method favoured for its strong robustness for DOA estimation. We consider a far-field point source detected by an arbitrary array with $\mathrm{N}$ sensors. The incident wave signal could be assumed as a plane wave when the source-receiver

Corresponding author: lihaipeng@hrbeu.edu.cn 
distance is large as compared with the array aperture size. The sensor array has a look direction $\left[\theta_{m}, \phi_{m}\right]$, where $\theta_{m}$ denotes the azimuth angle and $\phi_{m}$ represents the pitch angle. The basic model of the input signal is shown in Fig.1.

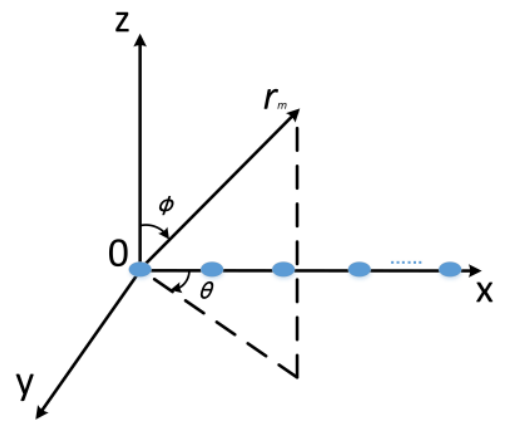

Fig. 1. Basic Model.

The output signal of the array is deduced by

$$
x_{m}(t)=\sum_{i=1}^{N}\left[s_{i}\left(t-\tau_{i, m}\right)+n_{i}(t)\right]
$$

where $s_{i}(t), i=1,2, \ldots, N$ is the incident signal, $n_{i}(t)$ is the noise vector, $\tau_{i, m}$ is the time delay of $i_{t h}$ sensor.

$$
\tau_{i, m}=-\mathbf{r}_{\mathbf{m}} \mathbf{p}_{\mathbf{i}} / c
$$

where $\mathbf{r}_{\mathbf{m}}$ is the look-direction dependent vector, $\mathbf{p}_{\mathbf{i}}$ is the coordinats of the receiver.

$$
\begin{gathered}
\mathbf{r}_{\mathbf{m}}=\left(\sin \phi_{m} \cos \theta_{m}, \sin \phi_{m} \sin \theta_{m}, \cos \phi_{m}\right) \\
\mathbf{p}_{\mathbf{i}}=\left(x_{i}, y_{i}, z_{i}\right)
\end{gathered}
$$

The DOA of the signal can be estimated by beam scanning, and the highest SNR will be achieved when the steering angle is aligned with the incident angle of the signal.

\section{Ray-tracing-based beamforming}

It should be noted that due to the curved wave propagation trajectory the DOA of signal may not be accurately estimated by using conventional beamforming method. To solve the above problem, one could use raytracing theory based on geometrical acoustics. The general expressions of the horizontal propagation distance and propagation time of sound are as follows.

$$
\begin{gathered}
r(z)=\int_{z_{0}}^{z} \frac{n c\left(z^{\prime}\right)}{\sqrt{1-n^{2} c^{2}\left(z^{\prime}\right)}} d z^{\prime} \\
\tau(z)=\int_{z_{0}}^{z} \frac{1}{c\left(z^{\prime}\right) \sqrt{1-n^{2} c^{2}\left(z^{\prime}\right)}} d z^{\prime} \\
n=\frac{\cos \left(\theta\left(z_{0}\right)\right)}{c\left(z_{0}\right)}=\frac{\cos \left(\theta\left(z_{1}\right)\right)}{c\left(z_{1}\right)}=\cdots=\frac{\cos \left(\theta\left(z_{n}\right)\right)}{c\left(z_{n}\right)}
\end{gathered}
$$

where $\mathrm{r}$ is the horizontal propagation distance; $\tau$ is the propagation time; $c\left(z^{\prime}\right)$ is the sound velocity at depth $z^{\prime}$; $n$ is Snell-satisfying ray-parameters, and $\theta\left(z_{i}\right)$ is the grazing angle at depth $z_{i}$.

The complex sound speed profile (SSP) is assumed to be a stratified medium with a constant sound speed gradient. The propagation time and horizontal distance are deduced by the following

$$
\begin{aligned}
& r=\sum_{i=0}^{N-1} \frac{c_{i}}{g_{i} \cos \theta_{i}}\left|\sin \theta_{i}-\sin \theta_{(i+1)}\right| \\
& \tau=\sum_{i=0}^{N-1} \frac{1}{2 g_{i}}\left|\ln \frac{1+\sin \theta_{i}}{1-\sin \theta_{i}}-\ln \frac{1+\sin \theta_{i+1}}{1-\sin \theta_{i+1}}\right|
\end{aligned}
$$

where $g_{i}$ is the gradient of each layer.

In this paper, we propose a ray-tracing-based beamforming method, which combines CBF with raytracing to obtain the advantages of each method, for UAPSs. We first apply CBF, which combines digital delay line with adaptive FIR filter to get precise spatial direction-of-arrival (DOA). Next, we apply the DOA as an initial grazing angle for ray-tracing to eliminate the curvature of the wave trajectory caused by the inhomogeneity of water, after that the position of target can be calculated with improved accuracy. The process of the proposed method is shown in Fig.2 and Fig.3.

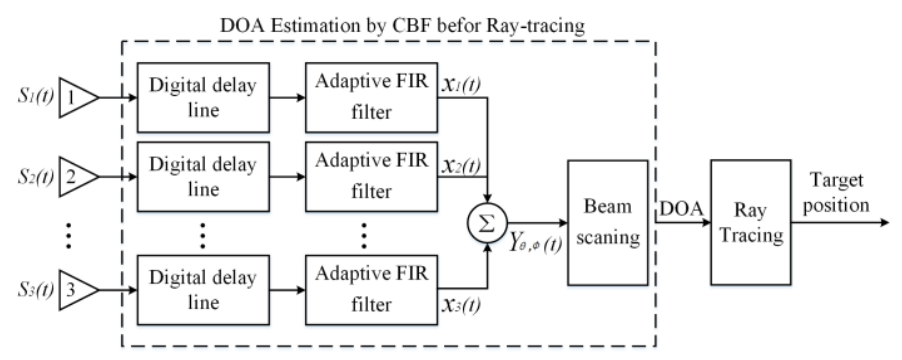

Fig. 2. Process of the proposed method.

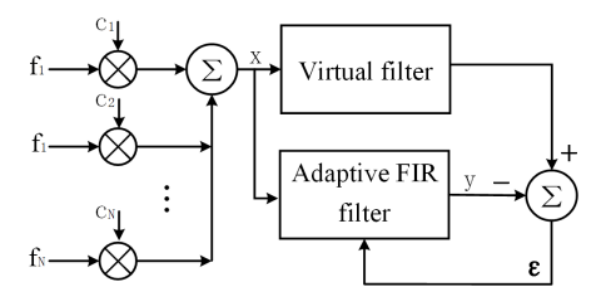

Fig. 3. Adaptive FIR filter.

\section{Test results}

\subsection{DOA estimation}

A pool test is conducted to verify our method. A 30elements uniform hexagonal array (Fig.4) and a sound source are fixed underwater, with a pitch angle of 45 and a azimuth angle of 187 (Fig.5). A linear frequency modulation (LFM) signal is used in this test, whose bandwidth is $9 \mathrm{kHz}-15 \mathrm{kHz}$ and pulse width is $25 \mathrm{~ms}$. The sound speed profile (SSP) of the pool is measured by a sound speed profiler (Fig.6). 

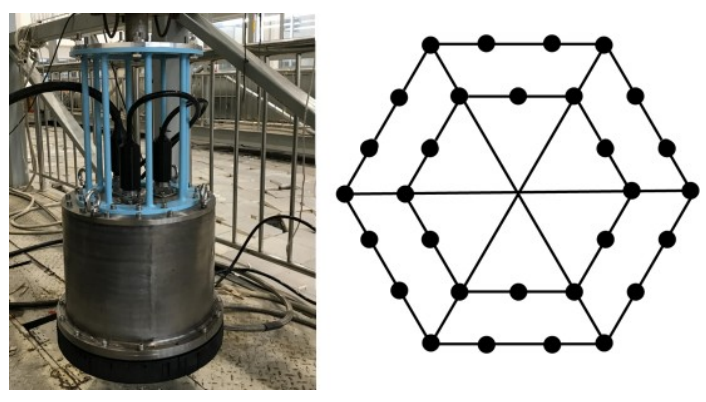

Fig. 4. 30-elements hexagonal planar array.

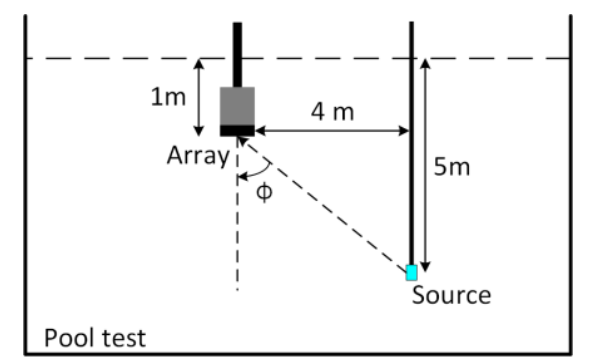

Fig. 5. The fixed system.

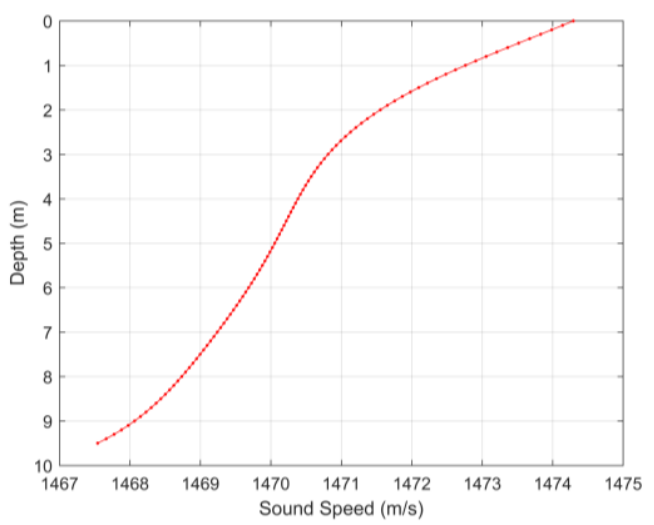

Fig. 6. Sound speed profile of the test pool.

CBF is used to get the DOA of the sound source by beam-scanning in the entire angular space. The spatial spectrum is shown in Fig.7. The SNR of the output signal of the array reaches the maximum value (Fig.8) when the main beam is aligned with the sound source.

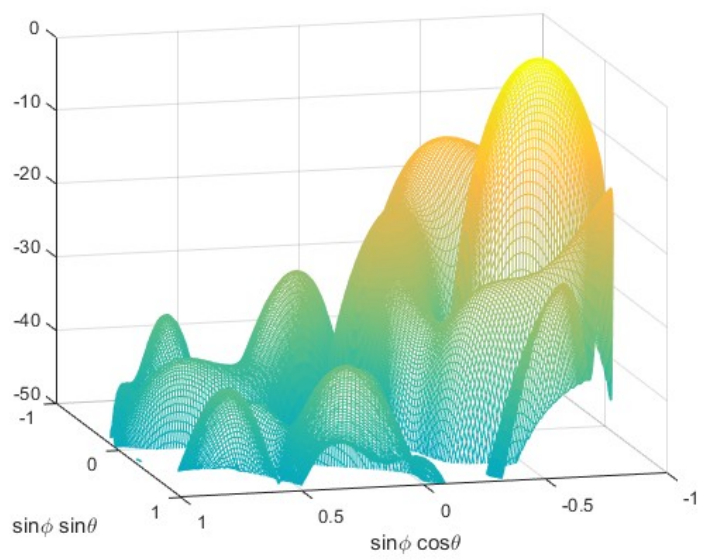

Fig. 7. Spatial spectrum of the input signals.

From Fig.7 we can calculate the pitch angle $\phi=49.01^{\circ}$ and the azimuth angle $\theta=187.6^{\circ}$ and think of them as the input value of ray-tracing. The comparison of the results is shown in Table 1, which show that the pitch angle calculated.

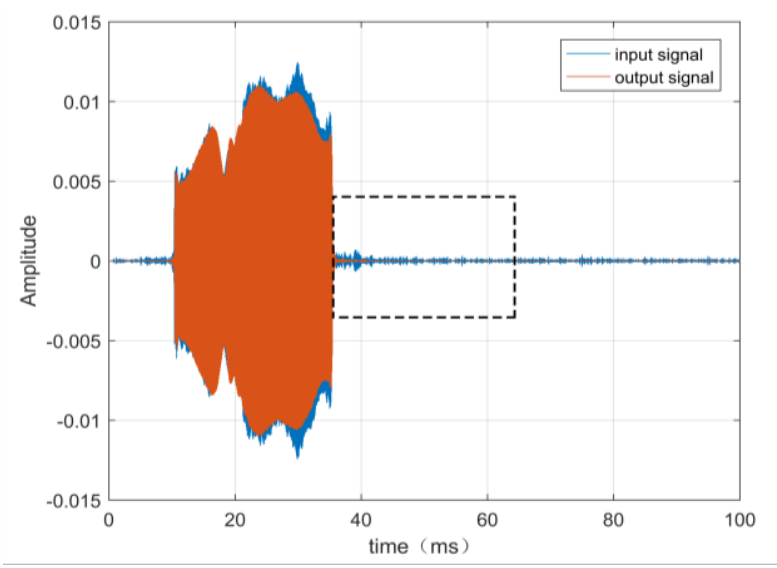

Fig. 8. Array input/output signal.

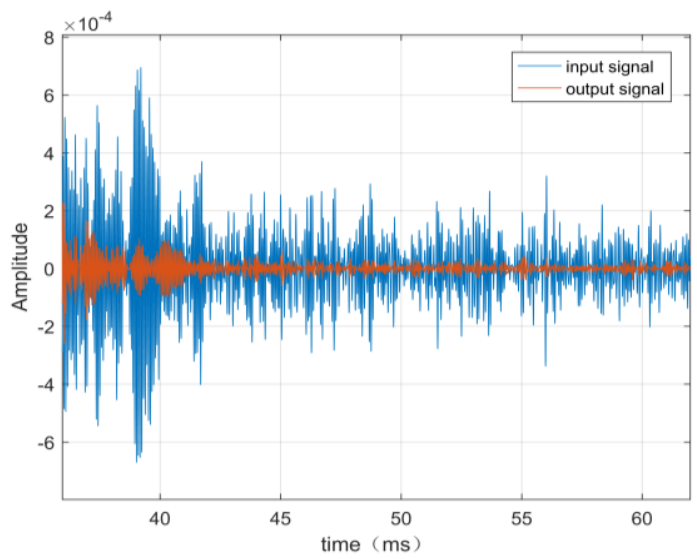

Fig. 9. Partial enlargement of Fig.8.

By CBF may not be well consistent with the true position of target because of the curved propagation effect. As a comparison, the Ray-tracing method solved this problem very well and reduced the horizontal positioning error $\left(\sqrt{\Delta x^{2}+\Delta y^{2}}\right)$ from $0.53 \mathrm{~m}$ to $0.13 \mathrm{~m}$ $(75 \%)$.

Table 1. Results comparison.

\begin{tabular}{|c|c|c|c|c|}
\hline Margin & $\phi\left(^{\circ}\right)$ & $\theta\left(^{\circ}\right)$ & $x(m)$ & $y(m)$ \\
\hline $\begin{array}{c}\text { Theoretical } \\
\text { value }\end{array}$ & 45.00 & 187.0 & 4.00 & 4.00 \\
\hline $\mathrm{CBF}$ & 49.01 & 187.6 & 4.53 & 3.94 \\
\hline $\begin{array}{c}\text { CBF\& } \\
\text { Ray-tracing }\end{array}$ & 46.20 & 187.6 & 4.13 & 3.97 \\
\hline
\end{tabular}

\subsection{Performance of the method in different SNR}

We further study the proposed method in different SNR conditions, whose performance is found to depend on the array gain of CBF. Here, the cross-correlation method is used for bearing estimation in UAPSs. We test the performance of the method by changing the SNR from $0 \mathrm{~dB}$ to $-15 \mathrm{~dB}$, and then verifying the correlation peak of input and output signals (Fig.10-Fig.13). 

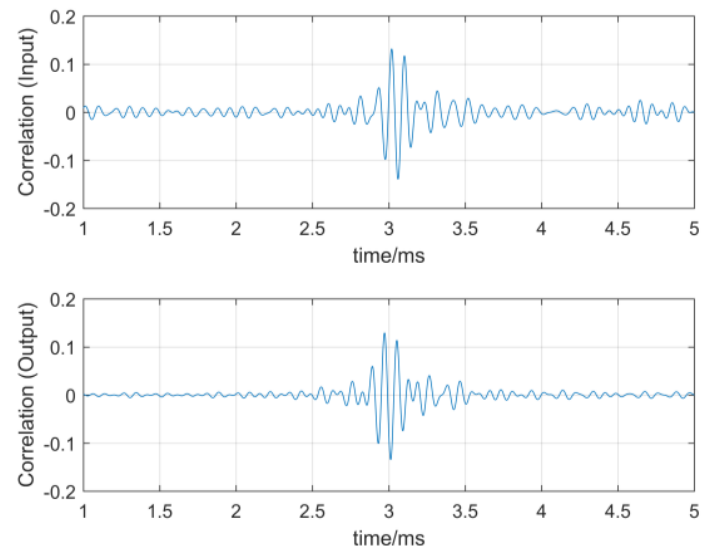

Fig. 10. Cross-correlation with $\mathrm{SNR}=0 \mathrm{~dB}$.
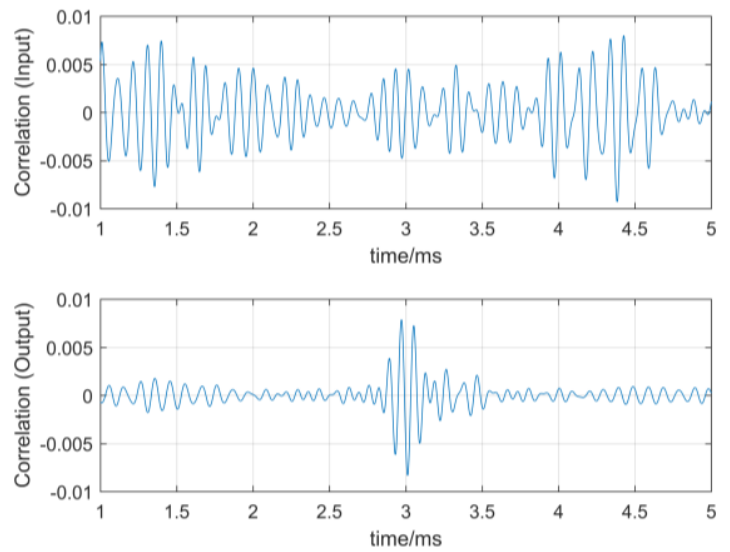

Fig. 11. Cross-correlation with $\mathrm{SNR}=-5 \mathrm{~dB}$.
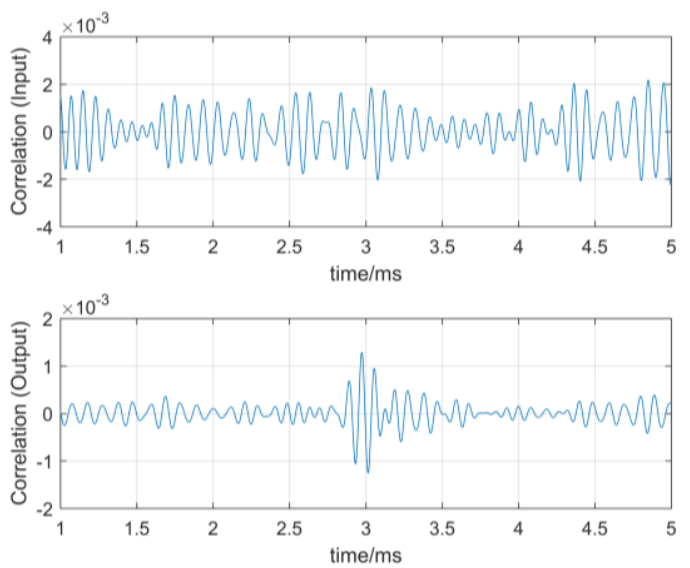

Fig. 12. Cross-correlation with $\mathrm{SNR}=-10 \mathrm{~dB}$.
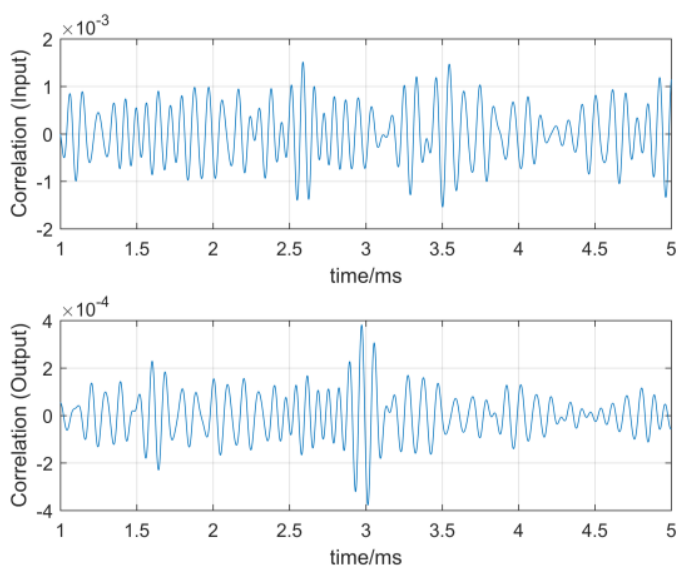

Fig. 13. Cross-correlation with $\mathrm{SNR}=-15 \mathrm{~dB}$.
It can be seen from the Fig.10-Fig.13 that the correlation peak of the input signal is week at $-5 \mathrm{~dB}$ SNR while the correlation peak of the output signal is obvious until -15dB SNR. The phenomenon is consistent with the array gain whose value is $10.25 \mathrm{~dB}$ (Fig.8).

\section{Conclusions}

In this paper, a ray-tracing based time-domain wideband fractional delay beamforming method for UAPSs is proposed. We take advantage of the array gain by $\mathrm{CBF}$ combining with digital delay lines and adaptive FIR filters. Ray-tracing is applied to improve positioning accuracy by eliminating the curvature of the trajectory caused by the inhomogeneity of water. The experimental results indicate that the method can effectively reduce the effect of ray bending and accurately estimate the position of target at low SNR.

This work was supported by the Key Program of the National Natural Science Foundation of China (Grant No.61531012).

\section{References}

1. R Urick, and J Robert. McGraw-Hill, 1983:1-100.

2. Y Han, C Zheng, D Sun. Ocean. Eng, 117(2016).

3. S Li, X Wang, L Zhang. IEEE J Oceanic Eng, 40(2015).

4. M Isik, and O Akan. IEEE Trans Commun, 8(2009):4457-4463.

5. L Liu, S Zhou, J Cui. Wire Commun Mob Com, 8(2010):977-994.

6. D Lambrinos, R Möller, T Labhart. Robo \& Auto Sys, 30(2000).

7. Z Li, S Dosso, D Sun. IEEE J Oceanic Eng, 41(2016).

8. A Douglass, H Song, D Dowling. J Acoust Soc Am, 142(2017).

9. T Yang. J Acoust Soc Am, 141(2017).

10. E Ruigrok, S Gibbons, $\mathrm{K}$ Wapenaar. J Seis, 21(2017).

11. P Ameer, L Jacob. IEEE Commun Lett, 14(2010), 930-932.

12. R Coates. Underwater Acoustic Systems. 1990:1100.

13. T Harold. J Vincent. J Acoust Soc Am, 102(1997), 3099-3100. 